\title{
Interacting with Music in a Social Setting
}

\author{
Ali Mazalek \\ Tangible Media Group \\ MIT Media Laboratory \\ 20 Ames St., Cambridge, MA 02139 USA \\ mazalek@media.mit.edu
}

\author{
Tristan Jehan \\ Hyperinstruments Group \\ MIT Media Laboratory \\ 20 Ames St., Cambridge, MA 02139 USA \\ tristan@media.mit.edu
}

\begin{abstract}
This paper describes the design of a new system for interacting with music in a social setting. MusiCocktail allows users to influence certain parameters of a precomposed and pre-recorded piece of music in the way they mix their beverages at a social gathering. This new form of interaction with music enables group participation in the creation of a rich musical environment.
\end{abstract}

\section{Keywords}

Social setting, interactive music, interface, mixing, drinks.

\section{INTRODUCTION}

Music has long been an important aspect of casual social environments. The collective experience of music in such environments not only creates a sense of community, but can also provide personal fulfillment for those involved [3]. For non-musicians however, these experiences and sensations are often limited by a passive consumption of music rather than an active participation in the musical setting.

We have designed an interactive system called MusiCocktail that adapts the ambient music at a social gathering depending on the way people mix their drinks. MusiCocktail can enhance the interaction in a social space by providing a new and entertaining form of interaction with music, by strengthening the sense of community through collaboration in a group setting, and by providing a reflection of ongoing activity in the environment. We give a conceptual and technical overview of the first implementation of our system, and discuss user feedback and future directions for the project.

\section{BACKGROUND}

By physically embodying music with fluids, the MusiCocktail system couples two common components of social events. This association is interesting since music is a time-based medium, and fluids (specifically drinks at a social gathering) have the unique property of changing over time as they are consumed and refilled. The interaction of mixing liquids becomes a metaphor for mixing music, and each mixed beverage becomes a physical representation of a particular stream or track of the ambient music being played. As the beverage is consumed, its associated stream of music slowly fades away. In this way, the music provides a sense of the ongoing activity and group presence, which is an important aspect of participating in a social milieu [2].

\section{RELATED WORK}

As early as $100 \mathrm{AD}$, the ancient Greeks used liquids to control musical instruments such as the water organ. Wateroperated automatons and water clocks were designed, respectively, to express motion and to measure time [1]. Like these systems, MusiCocktail uses fluids to represent a time-based medium (i.e. music).

More recently, the invention of the Jukebox gave people the option of selecting music in a social space. Also, Karaoke systems allow people to actively participate in the musical environment by singing along to pre-recorded music.

Tod Machover's group at the MIT Media Lab has done work that enables people to interact with music. For instance, the Singing Trees in the Brain Opera allow audience members to influence what they hear with the perceptual parameters of their voices, such as pitch and loudness [5].

The musicBottles project uses glass bottles as containers for music [4]. Though similar in concept, this idea differs from our MusiCocktail system in its metaphor and interaction style. The digital contents of the musicBottles have no physical representation and the interaction is focused on the opening and closing of bottles to release virtual contents.

\section{THE MUSICOCKTAIL SYSTEM}

The MusiCocktail system consists of two main components: the mixing station, and digitally augmented coasters that can be scattered around a social space.

At the mixing station, selecting a glass determines an instrument or track in the music that will be associated with that beverage. Adding different liquids to the glass mixes the musical parameters of that particular audio track. The quantity of each liquid determines the complexity of a musical parameter. Some parameters we have explored include richness, texture, rhythm, and brightness of the music. For instance, adding more of a particular liquid might make the music richer, meaning that it will have a greater number of notes and harmonies. As a user mixes their drink, the associated audio begins to play together with the already existing audio streams. The new stream 
will continue to play when the user removes their glass from the mixing station and brings it into the social space.

Digitally augmented coasters in the social area allow the system to keep track of the different audio streams that are currently playing (see Figure 2). The system updates its current state every time a glass is placed on a coaster. In this way, an audio stream can be slowly faded out as the level of liquid in the corresponding glass decreases.

\section{Technical Overview}

Our current implementation of the mixing station consists of two bottles (see Figure 1), allowing for the adjustment in real time of two parameters in each audio track. Force sensitive resistors (FSRs) detect how much liquid is being added to a glass by measuring the changes in weight.

In the social space, digitally enhanced coasters track the different glasses using tag-sensing technology. As in the mixing area, FSRs are used to determine the amount of liquid in each glass.

Currently, information is transmitted from the mixing station and coasters to the computer in real time through a serial connection. Eventually, this data transmission will be wireless. On the software side, a Java-based application controls both the audio mixing and playback.

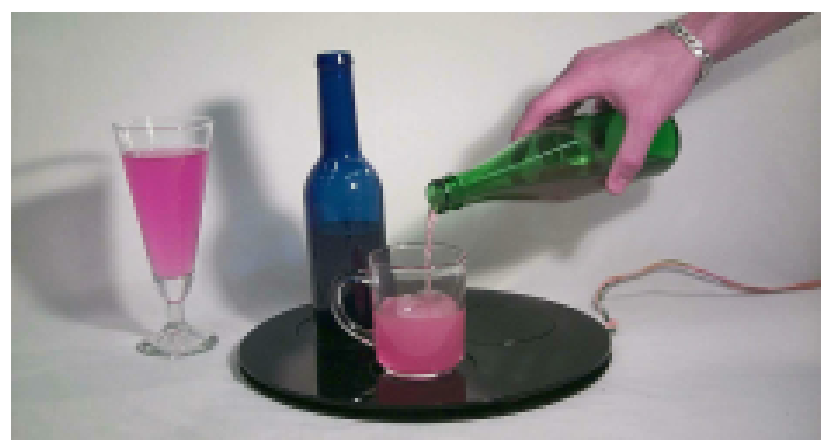

Figure 1: The mixing station with two modifier bottles.

\section{USER FEEDBACK}

Over a dozen users have experimented with our system informally, and initial feedback has been positive. Users liked the idea of being able to affect their musical environment by selecting different drinks. Several users expressed concerns that the beverage they selected (and liked) might be associated to an instrument or musical stream that they disliked. Other users like this aspect of the system and felt that it would incite them to try a greater variety of drinks simply to explore the musical contents of the system.

One user brought up an interesting point: it is often the case that when people are standing around and talking at a party, they like to hold their glass in their hand. If a user never places their glass on a coaster, the volume level of that audio track will never decrease, which could potentially lead to confusion (i.e. the music will continue to play when the content of the glass is gone). For this reason, it might be interesting to explore different ways of sensing the glasses and their contents that do not require use of digitally augmented coasters.

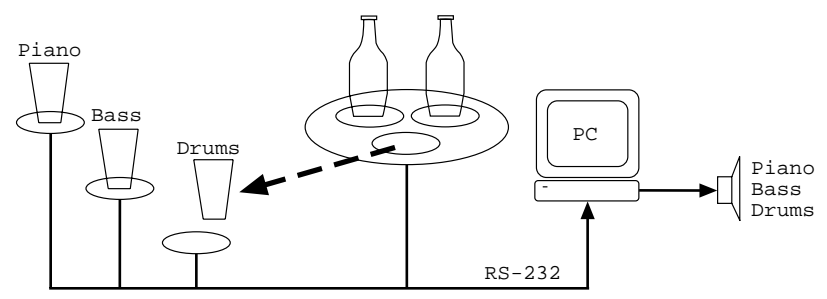

Figure 2: Diagram of the MusiCockatil system. Different beverages are associated with different tracks in the music.

\section{FUTURE WORK}

For the system to better fit standard social settings, the coasters and mixing area must transmit data to the computer wirelessly. The presence of a computer and wires in a social space is not aesthetically pleasing and creates unnecessary clutter. Ideally, the interface should be completely transparent: users should be able to go about their normal social activities without needing to know that their interactions with the ambient music are being mediated by a computer system.

Since our current implementation of the system has a limited selection of music (two compositions: jazz and techno), we would like to create a greater variety of musical contents. We would also like to perform more extended and formal user studies.

\section{CONCLUSIONS}

We are interested in the ways digital technology can enable non-musicians to interact with music in a social environment. We designed MusiCocktail to provide users with a means of collectively adjusting the parameters in a pre-composed and pre-recorded piece of music using an interaction that is familiar in social settings: mixing drinks. As a new form of interacting with music, we believe MusiCocktail has the potential to enhance social interactions and enable collaborative creation of a rich musical atmosphere.

\section{ACKNOWLEDGMENTS}

We thank Professor Hiroshi Ishii, Brygg Ullmer, Phil Frei, and the Tangible Interfaces class for their feedback. We also thank Dan Overholt for his help with the implementation.

\section{REFERENCES}

1. Bedini, Silvio A. The Role of Automata in the History of Technology. Technology Culture, v.5 no.1, Winter 1969.

2. Donath, Judith. Casual Collaboration. Proceedings of the International Conference on Multimedia Computing Systems (ICMCS), May 1994.

3. Frith, Simon. The Cultural Study of Popular Music. Cultural Studies, London, 1972, pp.174-182.

4. Ishii, H., et al. musicBottles. Conference Abstracts and Applications of SIGGRAPH '99. Emerging Technologies, (Los Angeles, California USA, August 8-13, 1999), ACM Press, pp.174.

5. Oliver, W. The Singing Tree: A Novel Interactive Musical Experience. Master's Thesis, MIT, 1997. 